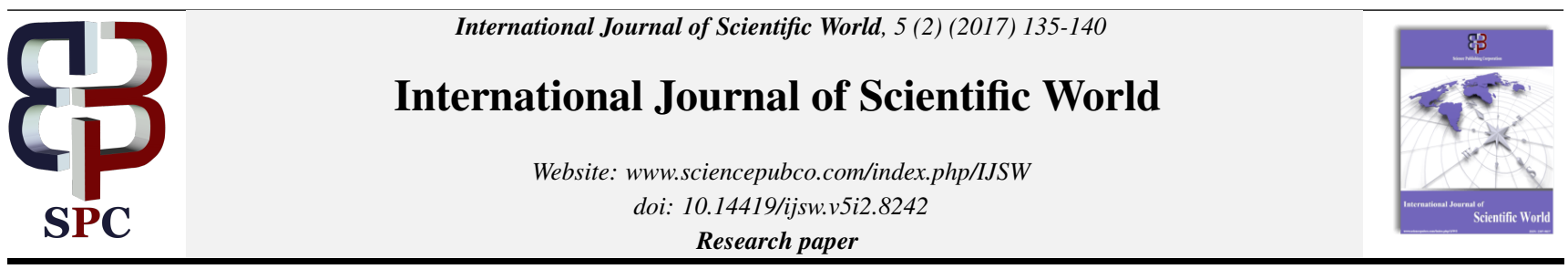

\title{
Bound lengths for Burr-XII distribution under step-stress PALT
}

\author{
Gyan Prakash \\ Department of Community Medicine, Moti Lal Nehru Medical College Allahabad, U. P., India. \\ Corresponding author E-mail: ggyanji@yahoo.com
}

\begin{abstract}
Some inferences based on Step-Stress Partially Accelerated Life Test (SS-PALT) are discussed in the present article. The Progressive Type-II censoring criterion with Random Removal scheme is used for determining the Approximate Confidence Lengths and One-Sample Bayes Prediction Bound Lengths for the unknown parameters of the Burr Type-XII distribution. Based on the simulated data, the analysis of the present discussion has been carried out.
\end{abstract}

Keywords: Partially Accelerated Life Test (PALT); Step-Stress Partially Accelerated Life Test (SS-PALT); Progressive Type-II censoring with Random Removal scheme (PRR-censoring); Approximate Confidence Length (ACL); One-Sample Bayes Prediction Bound Length (BPBL).

\section{Introduction}

The Burr Type-XII distribution was first introduced by [1]. Its probability density function and cumulative distribution are given below,

$f(x ; \theta, \sigma)=\sigma \theta x^{\sigma-1}\left(1+x^{\sigma}\right)^{-\theta-1} ; \theta>0, \sigma>0, x \geq 0$

and

$F(x ; \theta, \sigma)=1-\left(1+x^{\sigma}\right)^{-\theta} ; \theta>0, \sigma>0, x \geq 0$.

The twelve different types of cumulative distribution functions were included in the Burr system of distributions, that yield a variety of density shapes. The Burr Type-XII distribution has applied in several applied fields, including business, chemical engineering, quality control, medical, and reliability studies.

The failure rate function and the reliability functions of underlying distribution are given as

$\rho(x)=\sigma \theta \frac{x^{\sigma-1}}{1+x^{\sigma}} ; \theta>0, \sigma>0, x \geq 0$

$R(x)=\left(1+x^{\sigma}\right)^{-\theta} ; \theta>0, \sigma>0, x \geq 0$.

The shape of failure rate function $\rho(x)$ given in Eq. (3) does not affect by the parameter $\theta$. The failure rate function has a unimodal curve when $\sigma>1$ and it has decreasing failure rate function when $\sigma \leq 1$.
In the failure time modeling, quality control, and reliability studies, the present distribution is very valuable. Few important and recent references on the topic are included here. [2] deals with Bayesian inference and prediction problems of the Burr Type-XII distribution based on progressive first failure censored data. They present Bayesian inference under a squared error loss function by applying the Gibbs sampling procedure to draw Markov Chain Monte Carlo (MCMC) samples. [3] were discussed about the Bayes estimators for the parameters based on the upper records value.

[4] used a Koziol-Green model of random censorship for estimating the Bayes estimator of unknown parameters. [5] was discussed about the problem of estimating two shape parameters and the reliability function of Burr Type-XII distribution, based on a general progressively Type-II censored samples under Bayesian viewpoints. In addition, [6], [7], [8], and recently [9] also developed different inferences about the Burr Type-XII model.

[10] derived some inferences for the Burr Type-XII distribution under the Progressive Type-II censoring criterion. [11] also discussed the confidence limits for the underlying model based on Constant-Partially Accelerated Life Test (CP-ALT) under the Progressive Type-XII censoring with random removal. The focus of this study is to review and extend the results based on the Burr Type-II distribution under the Step-Stress partially accelerated lift test (SS-PALT). The censoring criterion has used here as the Progressive Type-II censoring with binomial removal. Based on SS-PALT, the approximate confidence lengths (ACL) and the One-Sample Bayes prediction bound lengths (BPBL) are obtained. The analysis of the present discussion has been carried out by a simulated data set. 


\section{Progressive Type-II Censoring With Ran- dom Removal}

Let us suppose an experiment in which $n$ independent and identical units $x_{1}, x_{2}, \ldots, x_{n}$ are placed on a life test at the beginning time and first $m ;(1 \leq m \leq n)$ failure items are observed. At the time of each failure occurring prior to the termination point, one or more surviving units are removed from the test. Experiment is terminated at the time of $m^{t h}$ failure, and all remaining live units are removed from the test. If $x_{(1)} \leq x_{(2)} \leq \ldots \leq x_{(m)}$ are the lifetimes of completely observed units to fail and $R_{1}, R_{2}, \ldots, R_{m} ;(m \leq n)$ are the numbers of units withdrawn at these failure times. Following [12], the joint probability density function under the Progressive Type-II censoring criterion is given as

$f(\theta, \sigma \mid \underline{x})=C_{p} \prod_{i=1}^{m} f\left(x_{i} ; \theta, \sigma\right)\left(1-F\left(x_{i} ; \theta, \sigma\right)\right)^{R_{i}}$.

Here, $f(\cdot)$ and $F(\cdot)$ be the probability density function and cumulative density function respectively given in Eq. (1) and Eq. (2) and $C_{p}$ is known as the progressive normalizing constant.

Let an individual unit being removed from the test at the $i^{\text {th }} ;(i=1,2, \ldots, m-1)$ failure is independent of the others with some probability $p$. Hence, $R_{i}$ of units removed at the $i^{t h} ;(i=1,2, \ldots, m-1)$ failure follows a Binomial distribution with parameters $p$ and $n-m-\sum_{j=1}^{i-1} r_{j}$. Following [11], $P(R)$ is now defined and obtained as

$$
\begin{gathered}
P(R)=P\left(R_{1}\right) P\left(R_{2} \mid R_{1}\right) \ldots P\left(R_{m-1} \mid R_{m-2}, \ldots, R_{1}\right) \\
=\Omega p^{a}(1-p)^{b} .
\end{gathered}
$$

where $\Omega=\frac{(n-m) !}{(n-m-a) !(a) !}, a=\sum_{j=1}^{m-1} R_{j}$ and $b=(m-1)(n-m)-$ $\sum_{j=1}^{m-1}(m-j) R_{j}$.

\section{Step-Stress Partially Accelerated Life Tests (SS-PALT)}

Accelerated Life Test (ALT) is used to control the reliability of a product in a spell by accelerating the practice environment. The acceleration factor is known or occurs a mathematical model that specifies the relationship between lifetime and stress. In some situations when the acceleration factor is unidentified or no any such models exist or are very hard to assume. The partially accelerated life tests (PALT) is a better option in such situations.

The stress can be applied in various ways, commonly used method is Step-Stress. In Step-Stress PALT, a test item is run first at normal use condition and, if it does not fail, then it is run at accelerated condition until failure occurs or the observation is censored. Several references have with SS-PALT, a little few of them are [13], [14], [15], [16], [17], [18] and [19].

Let us suppose, $n$ be the total test units are under the SSPALT, first run at the normal condition and if it does not fail by stress change time to $\varepsilon$, then the test is transformed to the accelerated condition and held until all units fail. [20] defined a tampered random variable model for the lifetime of a unit under SS-PALT and is given as

$X=\left\{\begin{array}{ccc}Y & ; & 0<Y \leq \varepsilon \\ \varepsilon+\frac{Y-\varepsilon}{\beta} & ; & Y>\varepsilon\end{array}\right.$ where $Y$ is the lifetime of a unit at normal condition, $\varepsilon$ is the stress change time and $\beta$ is the acceleration factor. Based on the tampered random variable (Eq. (7)) under the SS-PALT, the probability density function of the considered distribution is rewritten as

$f(x ; \theta, \sigma)=\left\{\begin{array}{lc}f_{1}=\sigma \theta x^{\sigma-1}\left(1+x^{\sigma}\right)^{-\theta-1} & ; \quad 0<x \leq \varepsilon \\ f_{2}=\sigma \beta \theta \tilde{x}^{\sigma-1}\left(1+\tilde{x}^{\sigma}\right)^{-\theta-1} & ; \quad x>\varepsilon ;\end{array}\right.$

where $\tilde{x}=(x-\varepsilon) \beta+\varepsilon$. Thus, the joint probability density (likelihood) function when parameter $\sigma$ is assume to be known, under SS-PALT based on PRR-censoring is defined and obtained as

$$
\begin{aligned}
L \propto \prod_{i=1}^{k}\left(f_{1}\left(1-F_{1}\right)^{R_{i}}\right) \times \prod_{i=k+1}^{m}\left(f_{2}\left(1-F_{2}\right)^{R_{i}}\right) \times P(R=r) \\
\propto \Omega p^{a}(1-p)^{b} \prod_{i=1}^{k} \sigma \theta x_{i}^{\sigma-1}\left(1+x_{i}^{\sigma}\right)^{-\theta R-1} \\
\quad \times \prod_{i=k+1}^{m}\left(\sigma \beta \theta W^{\sigma-1}(1+W)^{-\theta R-1}\right)
\end{aligned}
$$

$L \propto \theta^{m} \beta^{m-k} p^{a}(1-p)^{b} T_{0}(\underline{x}, \beta) e^{-\theta\left(T_{1}(\underline{x})+T_{2}(\underline{x}, \beta)\right)} ;$

where $T_{0}(\underline{x}, \beta)=\prod_{i=k+1}^{m}\left(\frac{W^{\sigma-1}}{1+W^{\sigma}}\right), T_{1}(\underline{x})=\sum_{i=1}^{k} R \log \left(1+x_{i}^{\sigma}\right)$, $T_{2}(\underline{x}, \beta)=\sum_{i=k+1}^{m} R \log \left(1+W^{\sigma}\right), \quad W=\left(x_{i}-\varepsilon\right) \beta+\varepsilon$ and $R=1+R_{i}$.

Taking logarithm of the likelihood function given in Eq. (8), we get

$$
\log L=m \log \theta+(m-k) \log \beta+a \log p
$$

$$
+b \log (1-p)+\log T_{0}(\underline{x}, \beta)-\theta T_{1}(\underline{x})-\theta T_{2}(\underline{x}, \beta) \text {. }
$$

\section{ML Based Approximate Confidence Lengths}

The first order derivative of the log-likelihood equation given in Eq. (9) with respect to the parameters $\theta, \beta$ and $p$ are obtained and given as

$$
\frac{\partial}{\partial \theta} \log L=\frac{m}{\theta}-\left(T_{1}(\underline{x})+T_{2}(\underline{x}, \beta)\right)
$$

$$
\frac{\partial}{\partial \beta} \log L=\frac{m-k}{\beta}-\sum_{i=k+1}^{m}\left\{\frac{\left(x_{i}-\varepsilon\right)\left((\sigma \theta R+1) W^{\sigma}-\sigma+1\right)}{\left(1+W^{\sigma}\right) W}\right\}
$$

and

$$
\frac{\partial}{\partial p} \log L=\frac{a}{p}-\frac{b}{1-p}
$$

Thus, the ML estimation corresponding to these parameters are obtained by solving following equations respectively

$$
\begin{aligned}
& \hat{\theta}_{M L}=\frac{m}{\sum_{i=1}^{k} R \log \left(1+x_{i}^{\sigma}\right)+\sum_{i=k+1}^{m} R \log \left(1+\hat{W}^{\sigma}\right)} \\
& (m-k)=\hat{\beta}_{M L} \sum_{i=k+1}^{m}\left\{\frac{\left(x_{i}-\varepsilon\right)\left(\left(\sigma \hat{\theta}_{M L} R+1\right) \hat{W}^{\sigma}-\sigma+1\right)}{\left(1+\hat{W}^{\sigma}\right) \hat{W}}\right\}
\end{aligned}
$$


and

$\hat{p}_{M L}=\frac{a}{a+b} ; \hat{X}=\left(x_{i}-\varepsilon\right) \hat{\beta}_{M L}+\varepsilon$

The close forms of these ML estimators are not possible. A numerical technique (Newton Raphson integral method) is applied here for obtaining the numerical findings of these ML estimators.

Based on the inverse of Fisher information matrix, the asymptotic variances and co-variances of ML estimator for the parameters are obtained

$\left[\begin{array}{ccc}-\frac{\partial^{2}}{\partial \theta^{2}} \log L & -\frac{\partial^{2}}{\partial \theta \partial \beta} \log L & -\frac{\partial^{2}}{\partial \theta \partial p} \log L \\ -\frac{\partial^{2}}{\partial \beta \partial \theta} \log L & -\frac{\partial^{2}}{\partial \beta^{2}} \log L & -\frac{\partial^{2}}{\partial \beta \partial p} \log L \\ -\frac{\partial^{2}}{\partial p \partial \theta} \log L & -\frac{\partial^{2}}{\partial p \partial \beta} \log L & -\frac{\partial^{2}}{\partial p^{2}} \log L\end{array}\right]_{\left(\hat{\theta}_{M L}, \hat{\beta}_{M L}, \hat{p}_{M L}\right)}^{-1}$

$=\left[\begin{array}{ccc}\operatorname{Var}\left(\hat{\theta}_{M L}\right) & \operatorname{Cov}\left(\hat{\theta}_{M L}, \hat{\beta}_{M L}\right) & \operatorname{Cov}\left(\hat{\theta}_{M L}, \hat{p}_{M L}\right) \\ \operatorname{Cov}\left(\hat{\beta}_{M L}, \hat{\theta}_{M L}\right) & \operatorname{Var}\left(\hat{\beta}_{M L}\right) & \operatorname{Cov}\left(\hat{\beta}_{M L}, \hat{p}_{M L}\right) \\ \operatorname{Cov}\left(\hat{p}_{M L}, \hat{\theta}_{M L}\right) & \operatorname{Cov}\left(\hat{p}_{M L}, \hat{\beta}_{M L}\right) & \operatorname{Var}\left(\hat{p}_{M L}\right)\end{array}\right]$

The second order derivatives of the log-likelihood equation are

$\frac{\partial^{2}}{\partial \theta^{2}} \log L=-\frac{m}{\theta^{2}}$

$\frac{\partial^{2}}{\partial \beta^{2}} \log L=-\frac{m-k}{\beta^{2}}-\sum_{i=k+1}^{m}\left\{\frac{\left(x_{i}-\varepsilon\right)^{2} W^{-2}}{1+W^{\sigma}}\right\} \times$

$\left\{\frac{\sigma^{2} \theta R+1}{W^{-\sigma}}-\right.$

$$
\left.-\frac{\left((\sigma \theta R+1) W^{\sigma}+1-\sigma\right)\left(1+W^{\sigma}(1+\sigma)\right)}{1+W^{\sigma}}\right\}
$$

$\frac{\partial^{2}}{\partial \beta^{2}} \log L=-\frac{a}{p^{2}}-\frac{b}{(1-p)^{2}}$

$\frac{\partial^{2}}{\partial \theta \partial \beta} \log L=\frac{\partial^{2}}{\partial \beta \partial \theta} \log L=-\sum_{i=k+1}^{m} R\left(x_{i}-\varepsilon\right)\left(\frac{\sigma W^{\sigma-1}}{1+W^{\sigma}}\right)$

$\frac{\partial^{2}}{\partial \theta \partial p} \log L=0=\frac{\partial^{2}}{\partial p \partial \theta} \log L$

and

$\frac{\partial^{2}}{\partial \beta \partial p} \log L=0=\frac{\partial^{2}}{\partial p \partial \beta} \log L$.

All the expressions of second order derivative involve the unknown parameters $\theta, \beta$ and $p$. Hence, the Fisher information matrix can be obtained by replacing the ML estimators of the parameters respectively. The applicability of normal approximation of ML Estimation is in small sample size. A log-transformation can be considered for improvements of the performance of the normal approximation.
Based on the normal approximation, the $(1-\tau) 100 \%$ approximate confidence intervals for the parameters $\theta, \beta$ and $p$ are obtained as

$$
\begin{gathered}
\left\{\hat{\theta}_{M L} \exp \left(\mp \frac{Z_{\tau / 2} \sqrt{\operatorname{Var}\left(\hat{\theta}_{M L}\right)}}{\hat{\theta}_{M L}}\right)\right\} \\
\left\{\hat{\beta}_{M L} \exp \left(\mp \frac{Z_{\tau / 2} \sqrt{\operatorname{Var}\left(\hat{\beta}_{M L}\right)}}{\hat{\beta}_{M L}}\right)\right\}
\end{gathered}
$$

and

$$
\left\{\hat{p}_{M L} \exp \left(\mp \frac{Z_{\tau / 2} \sqrt{\operatorname{Var}\left(\hat{p}_{M L}\right)}}{\hat{p}_{M L}}\right)\right\}
$$

Here, $Z_{\tau / 2}$ is the percentile of the standard normal distribution with right-tail probability $\tau / 2$.

\section{BPBL Under One-Sample Plan}

For the future observation $Y$, the Bayes predicative density is denoted by $h_{\Theta}(y \mid \underline{x})$ and obtained by simplifying following expression

$h_{\Theta}(y \mid \underline{x}) \propto \int_{\Theta} f(y ; \theta, \sigma) \pi_{\Theta}^{*} d \Theta ;$

where $\pi_{\Theta}^{*} ;(\Theta=\theta, \beta, p)$ be the posterior density corresponding to the parameter $\Theta(=\theta, \beta, p)$ respectively.

A conjugate family of prior, two-parameter Gamma distribution is considered as the prior distribution for the shape parameter $\theta$, having probability density

$\pi_{\theta} \propto \theta^{\alpha-1} e^{-\theta} ; \alpha>0, \theta>0$.

For the acceleration factor $\beta$, the vague prior is assumed, so that the prior does not have any significant role in the analyses that follow. The prior probability density is

$\pi_{\beta} \propto \beta^{-1} ; \beta>0$

The prior distribution regarding the factor $p$ is assumed as Beta distribution, with probability density

$\pi_{p} \propto p^{\gamma-1}(1-p)^{\lambda-1} ; \gamma>0, \lambda>0,0 \leq p \leq 1$.

Thus the joint prior distribution for $\Theta(=\theta, \beta, p)$ is now given as

$\pi_{(\theta, \beta, p)} \propto \theta^{\alpha-1} e^{-\theta} \beta^{-1} p^{\gamma-1}(1-p)^{\lambda-1}$.

Therefore, the joint and marginal posterior densities for the parameters are obtained respectively as

$$
\begin{array}{r}
\pi_{(\theta, \beta, p)}^{*}=\frac{\pi_{(\theta, \beta, p)} \times L}{\int_{\beta} \int_{\theta} \int_{p} \pi_{(\theta, \beta, p)} \times L d \theta d p d \beta} \\
\propto \frac{\theta^{m+\alpha-1} \beta^{m-k-1} T_{0}(\underline{x}, \beta) e^{-\theta Z_{\beta}}}{\int_{\beta} \beta^{m-k-1} T_{0}(\underline{x}, \beta) \int_{\theta} \theta^{m+\alpha-1} e^{-\theta Z_{\beta}} d \theta d \beta} \\
\times \frac{p^{a+\gamma-1}(1-p)^{b+\lambda-1}}{\int_{p} p^{a+\gamma-1}(1-p)^{b+\lambda-1} d p}
\end{array}
$$




$$
\begin{aligned}
\pi_{(\theta, \beta, p)}^{*} \propto \Omega \theta^{m+\alpha-1} \beta^{m-k-1} & T_{0}(\underline{x}, \beta) \\
& \times e^{-\theta Z_{\beta}} p^{a+\gamma-1}(1-p)^{b+\lambda-1}
\end{aligned}
$$

and the marginal posteriors are

$\pi_{\theta}^{*} \propto \frac{\Omega B(a+\gamma, b+\lambda)}{\theta^{-m-\alpha+1}} \int_{\beta} \beta^{m-k-1} T_{0}(\underline{x}, \beta) e^{-\theta Z_{\beta}} d \beta$

$\pi_{\beta}^{*} \propto \Omega B(a+\gamma, b+\lambda) \Gamma(m+\alpha) \beta^{m-k-1} \frac{T_{0}(\underline{x}, \beta)}{Z_{\beta}^{m+\alpha}}$

and

$\pi_{p}^{*} \propto \Omega \Gamma(m+\alpha) \frac{p^{a+\gamma-1}}{(1-p)^{-b-\lambda+1}} \int_{\beta} \beta^{m-k-1} \frac{T_{0}(\underline{x}, \beta)}{Z_{\beta}^{m+\alpha}} d \beta$

where $\Omega=\left\{B(a+\gamma, b+\lambda) \Gamma(m+\alpha) \int_{\beta} \beta^{m-k-1} \frac{T_{0}(\underline{x}, \beta)}{Z_{\beta}^{m+\alpha}} d \beta\right\}^{-1}$ and $Z_{\beta}=T_{1}(\underline{x})+T_{2}(\underline{x}, \beta)+1$.

Using Eq. (1) and Eq. (15) in Eq. (14), the Bayes predictive density of future variable $Y$, for the parameter $\theta$ is obtained as

$$
\begin{aligned}
h_{\theta}(y \mid \underline{x})= & \int_{\theta} f(y ; \theta, \sigma) \times \pi_{\theta}^{*} d \theta \\
= & \Omega B(a+\gamma, b+\lambda) \frac{\sigma y^{\sigma-1}}{1+y^{\sigma}} \int_{\beta} \beta^{m-k-1} T_{0}(\underline{x}, \beta) \\
& \times \int_{\theta} \theta^{m+\alpha} \exp \left(-\theta\left(Z_{\beta}+\log \left(1+y^{\sigma}\right)\right)\right) d \theta d \beta \\
\Rightarrow h_{\theta}(y \mid \underline{x})= & \Omega_{\theta}^{*} \frac{\sigma y^{\sigma-1}}{1+y^{\sigma}} \int_{\beta} \beta^{m-k-1} \\
& \times \frac{T_{0}(\underline{x}, \beta)}{\left(Z_{\beta}+\log \left(1+y^{\sigma}\right)\right)^{m+\alpha+1}} d \beta
\end{aligned}
$$

where $\Omega_{\theta}^{*}=\Omega B(a+\gamma, b+\lambda) \Gamma(m+\alpha+1)$.

let $l_{1}$ and $l_{2}$ be the lower and upper Bayes prediction limits for the future observation $Y$ and $(1-\tau)$ be the confidence prediction coefficient, then the one-sided Bayes prediction bound limits are obtain by solving following equality

$\operatorname{Pr}\left(Y \leq l_{1}\right)=\frac{\tau}{2}=\operatorname{Pr}\left(Y \geq l_{2}\right)$.

Using Eq. (18) \& Eq. (19), the Bayes predictive bound limits for the parameter $\theta$ are obtained by solving following equations

$$
\Lambda_{1}=\int_{\beta} \beta^{m-k-1} \frac{T_{0}(\underline{x}, \beta)}{Z_{\beta}^{m+\alpha}}\left\{1-\left(1+\frac{\log \left(1+l_{1}^{\sigma}\right)}{Z_{\beta}}\right)^{-m-\alpha}\right\} d \beta
$$

and

$$
\Lambda_{2}=\int_{\beta} \beta^{m-k-1} \frac{T_{0}(\underline{x}, \beta)}{Z_{\beta}^{m+\alpha}}\left\{1-\left(1+\frac{\log \left(1+l_{2}^{\sigma}\right)}{Z_{\beta}}\right)^{-m-\alpha}\right\} d \beta
$$

where $\Lambda_{1}=\frac{\tau}{2 \Omega B(a+\gamma, b+\lambda) \Gamma(m+\alpha)}$ and $\Lambda_{2}=\left(\frac{2-\tau}{\tau}\right) \Lambda_{1}$.

An analytical solution of the expressions Eq. (20) and Eq. (21) do not exists. A numerical technique is applied here for the numerical findings of the prediction limits. Based on numerical findings of $l_{1}$ from Eq. (20) and $l_{2}$ from Eq. (21), the Bayes predictive bound length (BPBL) for the parameter $\theta$ is obtained as

$L_{\theta}=l_{2}-l_{1}$.

Similarly, the Bayes predictive density and prediction limits for the parameter $\beta$ are obtained by solving following equations respectively;

$h_{\beta}(y \mid \underline{x})=\Omega B(a+\gamma, b+\lambda) \Gamma(m+\alpha) \sigma \theta$

$$
\begin{aligned}
& \times \int_{\beta} \beta^{m-k} \frac{T_{0}(\underline{x}, \beta)}{Z_{\beta}^{m+\alpha}}((y-\varepsilon) \beta+\varepsilon)^{\sigma-1} \\
& \times\left(1+((y-\varepsilon) \beta+\varepsilon)^{\sigma}\right)^{-\theta-1} d \beta
\end{aligned}
$$

$\Lambda_{1}=\int_{\beta} \beta^{m-k-1} \frac{T_{0}(\underline{x}, \beta)}{Z_{\beta}^{m+\alpha}}\left(Z_{1}-Z_{2}\right) d \beta$

and

$\Lambda_{2}=\int_{\beta} \beta^{m-k-1} \frac{T_{0}(\underline{x}, \beta)}{Z_{\beta}^{m+\alpha}}\left(Z_{1}-Z_{3}\right) d \beta$

where $Z_{1}=1+(\varepsilon-\varepsilon \beta)^{\sigma}, Z_{2}=1+\left(\varepsilon+\left(l_{1}-\varepsilon\right) \beta\right)^{\sigma}$ and $Z_{3}=$ $1+\left(\varepsilon+\left(l_{2}-\varepsilon\right) \beta\right)^{\sigma}$.

Based on the numerical findings of the lower and upper limits, the BPBL for the parameter $\beta$ is obtained by the relation

$L_{\beta}=l_{2}-l_{1}$

Now, the Bayes predictive density for $Y$, prediction limits and resultant BPBL corresponding to the parameter $p$, are obtained by solving following equalities

$h_{p}(y \mid \underline{x})=\Omega_{p}^{*} \sigma \theta \frac{y^{\sigma-1}}{\left(1+y^{\sigma}\right)^{\theta+1}}$,

$l_{1}=\left\{\left(1-\frac{\varepsilon}{2 \Omega_{p}^{*}}\right)^{-\frac{1}{\theta}}-1\right\}^{\frac{1}{\sigma}}$,

$l_{2}=\left\{\left(1-\frac{2-\varepsilon}{2 \Omega_{p}^{*}}\right)^{-\frac{1}{\theta}}-1\right\}^{\frac{1}{\sigma}}$

and

$L_{p}=l_{2}-l_{1}$

where $\Omega_{p}^{*}=\Omega B(a+\gamma, b+\lambda) \Gamma(m+\alpha) \int_{\beta} \beta^{m-k-1} \frac{T_{0}(\underline{x}, \beta)}{Z_{\beta}^{m+\alpha}} d \beta$. 


\section{Numerical Illustration}

\subsection{Estimation of Optimal Stress Change Time}

The optimal stress change time $\varepsilon^{*}$ is obtained by minimizing the asymptotic variance of the Maximum Likelihood estimation of the parameters $\theta, p$ and the acceleration factor $\beta$. The asymptotic variances of $\hat{\theta}_{M L}, \hat{\beta}_{M L}$ and $\hat{p}_{M L}$ are given by the diagonal elements of the inverse of the Fisher information matrix given in Eq. (10). The optimal stress change time $\varepsilon^{*}$ of $\varepsilon$ is obtained by minimizing the generalized asymptotic variance of the MLE of the model parameters and the acceleration factor. Using Mathematica 7.00, the optimal stress change time $\varepsilon^{*}$ is 2.169 for $\theta=0.25, \sigma=0.25, \beta=1.20$, $n=30$ and $m=20$.

\subsection{Simulation Study}

In the present section, a complete numerical illustration has presented for the studying the properties of the present inference by the help of simulated data. A set of 10,000 random sample generated, each of size $n=30$ by using following relation

$x_{i}=\left\{\left(1-U_{i}\right)^{-\frac{1}{\theta}}\right\}^{\frac{1}{\sigma}}$

with the help of $(\theta, \sigma)(=0.25,0.50,1.00)$. Here, $U_{i}$ are independently distributed $U(0,1)$. The progressive censoring pattern for $m=20$ is considered as $R_{i}(=1,0,2,0,0,3,0,1,2,0,4,0,0,5,2,2,0,1,0,2)$. The ML estimate and corresponding mean square error (MSE) are given in the Table 1.

It is observed from the table is that; the ML estimate and their corresponding mean square errors are increasing as the combination of the model parameters increase. A similar trend has also seen when the optimal stress change time $\varepsilon^{*}$ varies with the model parameters.

A log-transformed ACI and BPBL for all the parameters are obtained and given in Table $2 \&$ Table 3 for pre-selected values as discussed above respectively. It is observed from the table is that, the Approximate Confidence Length (ACL) increases when $\tau$ increases. ACL decrease first and then increases when model parametric value increases. A similar trend has also seen when, $\varepsilon^{*}$ varies. All properties regarding the ACL are similar explainable for BPBL. However, the magnitude of the BPBL is robust when compared with ACL.

\section{References}

[1] Burr, W. I. 1942. Cumulative frequency distribution, Annals of Mathematical Statistics, 13, 215 - 232.

[2] Soliman, A. A., Abd-Ellah, A. H., Abou-Elheggag, N. A. \& Modhesh, A. A. 2011. Bayesian inference and prediction of Burr Type - XII distribution for progressive first failure censored sampling. Intelligent Information Management, 3, 175 - 185.

[3] Nadar, M. \& Papadopoulos, A. S. (2011). Bayesian analysis for the Burr Type - XII distribution based on record values, Statistica, 71 (4), $421-435$.

[4] Danish, M. Y. \& Aslam, M. 2014. Bayesian analysis of censored Burr-XII distribution. Electronic Journal of Applied Statistical Analysis, 7 (2), 326 - 342.

[5] Jang, D.-H., Jung, M., Park, J.-H., \& Kim, C. 2014. Bayesian estimation of Burr Type-XII distribution based on general Progressive Type - II censoring. Applied Mathematical Sciences, 8 (69), 3435 3448.

[6] Li, X., Shi, Y., Wei, J., \& Chai, J. 2007. Empirical Bayes estimators of reliability performances using LINEX loss under progressively Type II censored samples. Mathematics and Computers in Simulation, 73 (5), 320 - 326.
[7] Lee, W. C., Wu, J. W. \& Hong, C. W. 2009. Assessing the lifetime performance index of products from progressively Type - II right censored data using Burr - XII model. Mathematics and Computers in Simulation, 79 (7), 2167 - 2179

[8] Al-Hussaini, E. K. \& Hussein, M. 2011. Estimation using censored data from exponentiated Burr Type - XII population. American Open Journal of Statistics, 1, 33 - 45.

[9] Rao, G. S., Aslam, M. \& Kundu, D. 2015. Burr - XII distribution parametric estimation and estimation of reliability of multicomponent stress-strength. Communications in Statistics - Theory and Methods, 44, 4953 - 4961.

[10] Prakash, G. 2017 a. Progressive censored Burr Type - XII distribution under random removal scheme: Some inferences. Afrika Statistika, 12 (2), $1273-1284$.

[11] Prakash, G. 2017 b. Confidence limits for progressive censored Burr Type - XII data under Constant-Partially ALT. Journal of Statistics Applications \& Probability, 6 (2), 295 - 303.

[12] Prakash, G. 2015. A comparative study based on Bayes estimation under different censoring criterion. Journal of Data Science, 13 (2), $261-280$.

[13] Bhattacharyya, G. K. \& Soejoeti, Z. 1989. A tampered failure rate model for step-stress accelerated life test. Communication in Statistics Theory \& Method, 18, 1627 - 1643.

[14] Abdel-Hamid, A. H. \& Al-Hussaini, E. K. 2007. Progressive stress accelerated life tests under finite mixture models. Metrika, 66, 213 231.

[15] Ismail, A. A. \& Aly, H. M. 2009. Optimal planning of failure-step stress partially accelerated life tests under Type - II Censoring. International Journal of Mathematical Analysis, 3 (31), 1509 - 1523.

[16] Srivastava, P. W. \& Mittal, N. 2010. Optimum step-stress partially accelerated life tests for the truncated logistic distribution with censoring. Applied Mathematical Modelling, 34, 3166 - 3178.

[17] Tangi, Y., Guani, Q., Xu, P., \& Xu, H. 2012. Optimum design for Type - I step-stress accelerated life tests of two-parameter Weibull distributions. Communication in Statistics - Theory and Methods, 41, $3863-3877$.

[18] Hyun, S. \& Lee, J. 2015. Constant-stress partially accelerated life testing for Log-Logistic distribution with censored data. Journal of Statistics Applications and Probability, 4 (2), 293 - 201.

[19] Abdel-Hamid, A. H. 2016. Estimations in step-partially accelerated life tests for an Exponential lifetime model under Progressive Type-I censoring and general entropy loss function. Journal of Mathematics and Statistical Science, 2016, 75 - 93.

[20] DeGroot, M. H. \& Goel, P. K. 1979. Bayesian and optimal design in partially accelerated life testing. Naval Research Logistics, 16(2), 223 235 .

Table 1: ML Estimate and Mean Square Error

\begin{tabular}{c|c|c|c|c}
\hline$(\theta, \sigma)$ & $\varepsilon^{*}$ & $\hat{\theta}_{M L}$ & $\hat{\beta}_{M L}$ & $\hat{p}_{M L}$ \\
\hline$(0.25,0.25)$ & 2.169 & 1.0031 & 1.0197 & 0.9657 \\
\hline$(0.50,0.50)$ & 2.341 & 1.0109 & 1.0387 & 0.9703 \\
\hline$(1.00,1.00)$ & 2.398 & 1.0197 & 1.0531 & 0.9791 \\
\hline$(\theta, \sigma)$ & $\varepsilon^{*}$ & $R\left(\hat{\theta}_{M L}\right)$ & $R\left(\hat{\beta}_{M L}\right)$ & $R\left(\hat{p}_{M L}\right)$ \\
\hline$(0.25,0.25)$ & 2.169 & 0.6789 & 0.7893 & 0.6891 \\
\hline$(0.50,0.50)$ & 2.341 & 0.6809 & 0.8001 & 0.6902 \\
\hline$(1.00,1.00)$ & 2.398 & 0.6897 & 0.8309 & 0.7004 \\
\hline
\end{tabular}

Table 2: Log-Transformed Approximate Confidence Length

\begin{tabular}{c|c|c|c|c|c}
\hline & $(\theta, \sigma)$ & $\varepsilon^{*}$ & $\tau=99 \%$ & $\tau=95 \%$ & $\tau=90 \%$ \\
\hline \multirow{2}{*}{$\theta$} & $0.25,0.25$ & 2.169 & 1.5051 & 1.4416 & 1.3808 \\
\cline { 2 - 6 } & $0.50,0.50$ & 2.341 & 1.0349 & 0.9913 & 0.9495 \\
\cline { 2 - 6 } & $1.00,1.00$ & 2.398 & 1.0491 & 1.0049 & 0.9625 \\
\hline \multirow{2}{*}{$\beta$} & $0.25,0.25$ & 2.169 & 1.4513 & 1.3701 & 1.3415 \\
\cline { 2 - 6 } & $0.50,0.50$ & 2.341 & 1.0234 & 0.9702 & 0.9689 \\
\cline { 2 - 6 } & $1.00,1.00$ & 2.398 & 1.0357 & 1.0099 & 0.9802 \\
\hline \multirow{3}{*}{$p$} & $0.25,0.25$ & 2.169 & 1.6701 & 1.5197 & 1.4322 \\
\cline { 2 - 6 } & $0.50,0.50$ & 2.341 & 1.0197 & 1.0067 & 0.9555 \\
\cline { 2 - 6 } & $1.00,1.00$ & 2.398 & 1.3407 & 1.2842 & 1.2301 \\
\hline
\end{tabular}


Table 3: Bayes Prediction Bound Lengths (BPBL)

\begin{tabular}{c|c|c|c|c|c}
\hline & $(\theta, \sigma)$ & $\varepsilon^{*}$ & $\tau=99 \%$ & $\tau=95 \%$ & $\tau=90 \%$ \\
\hline \multirow{3}{*}{$\theta$} & $0.25,0.25$ & 2.169 & 1.4584 & 1.3965 & 1.3171 \\
\cline { 2 - 6 } & $0.50,0.50$ & 2.341 & 1.0197 & 0.9771 & 0.9464 \\
\cline { 2 - 6 } & $1.00,1.00$ & 2.398 & 1.0335 & 0.9984 & 0.9569 \\
\hline \multirow{3}{*}{$\beta$} & $0.25,0.25$ & 2.169 & 1.4059 & 1.3167 & 1.2308 \\
\cline { 2 - 6 } & $0.50,0.50$ & 2.341 & 0.9185 & 0.8696 & 0.8453 \\
\cline { 2 - 6 } & $1.00,1.00$ & 2.398 & 0.9905 & 0.9653 & 0.9263 \\
\hline \multirow{3}{*}{$p$} & $0.25,0.25$ & 2.169 & 1.5294 & 1.4227 & 1.0373 \\
\cline { 2 - 6 } & $0.50,0.50$ & 2.341 & 0.9548 & 0.9322 & 0.8702 \\
\cline { 2 - 6 } & $1.00,1.00$ & 2.398 & 1.1308 & 1.0229 & 0.9172 \\
\hline
\end{tabular}

